\title{
Elevated preoperative CEA is associated with worse survival in stage I-III rectal cancer patients
}

\author{
I Tarantino*,1,5, R Warschkow ${ }^{1,2,5}$, M Worni $^{3}, K_{\text {Merati-Kashani', D Köberle }}^{4}$, BM Schmied', SA Müller', \\ T Steffen', T Cerny ${ }^{4}$ and U Güller ${ }^{3,4}$
}

'Department of Surgery, Kantonsspital St Gallen, 9007 St Gallen, Switzerland; ${ }^{2}$ Institute of Medical Biometry and Informatics, University of Heidelberg, 69120, Heidelberg, Germany; ${ }^{3}$ University Clinics for Visceral Surgery and Medicine, University Hospital Berne, 3010 Berne, Switzerland; ${ }^{4}$ Department of Medical Oncology and Hematology, Kantonsspital St Gallen, 9007 St Gallen, Switzerland

BACKGROUND: The objective of this investigation was to assess whether preoperative carcinoembryonic antigen (CEA) level is an independent predictor of overall survival in rectal cancer patients.

METHODS: All patients $(n=504)$ undergoing a resection for stage I-III rectal cancer at the Kantonsspital St Gallen were included into a database between 199I and 2008. The impact of preoperative CEA level on overall survival was assessed using risk-adjusted Cox proportional hazard regression models and propensity score methods.

RESULTS: In risk-adjusted Cox proportional hazard regression analyses, preoperative CEA level (hazard ratio (HR): I.98, 95\% confidence interval $(\mathrm{Cl})$ : I.36-2.90, $P<0.00 \mathrm{I})$, distance from anal verge $(<5 \mathrm{~cm}$ : HR: I.93, 95\% Cl: I.II -3.37 ; $P=0.039)$, older age (HR: I.07, 95\% Cl: I.05-I.09; P<0.00I), lower body mass index (HR: 0.94, 95\% Cl: 0.89-0.98; $P=0.006)$, advanced tumour stage (stage II HR: I.4I, 95\% Cl: 0.85-2.32; stage III HR: 2.08, 95\% Cl: I.3I-3.3।; P=0.004), R I resection (HR: 5.65, 95\% Cl: I.59-20.I; $P=0.005)$ and chronic kidney disease (HR: $2.28,95 \% \mathrm{Cl}: 1.03-5.04 ; P=0.049$ ) were all predictors for poor overall survival.

CONCLUSION: This is one of the first investigations based on a large cohort of exclusively rectal cancer patients demonstrating that preoperative CEA level is a strong predictor of decreased overall survival. Preoperative CEA should be used as a prognostic factor in the preoperative assessment of rectal cancer patients.

British Journal of Cancer (2012) 107, 266-274. doi:I0.1038/bjc.2012.267 www.bjcancer.com

Published online 26 June 2012

(C) 2012 Cancer Research UK

Keywords: CEA; rectal cancer; overall survival

Colorectal cancer is the malignancy with the fourth highest prevalence among females and males in the Western world with a lifetime risk of 1 in 20 persons (Parkin et al, 2005; CancerStatistics, 2010; Howlader et al, 2011). Among colorectal cancer patients, about $30 \%$ have a cancer of the rectum. Currently, the most important prognostic factor determining treatment strategy and outcomes in rectal cancer patients is tumour stage; however, the sole use of tumour stage to predict prognosis is imperfect.

Before rectal cancer resection, the guidelines of the National Comprehensive Cancer Network (NCCN) suggest routine measurement of preoperative carcinoembryonic antigen (CEA), mainly for monitoring postoperative surveillance (NCCN, 2012). However, there is persistent debate about the impact of an increased preoperative CEA as an independent predictive tumour marker in patients with rectal cancer.

Over 40 years ago, CEA was first described by Gold and Freedman and was subsequently identified as a prognostic tumour marker for colorectal cancer (Wanebo et al, 1978). Since then, postoperative measurement of CEA is an established tool in the follow-up surveillance of colorectal cancer patients (Kim et al, 2009; Lin et al,

\footnotetext{
*Correspondence: Dr I Tarantino; E-mail: Ignazio.Tarantino@kssg.ch

${ }^{5}$ These authors contributed equally to this work.

Received 20 March 2012; revised I4 May 2012; accepted 18 May 2012; published online 26 June 2012
}

2011; NCCN, 2012). Increased early postoperative CEA levels are often a sign of remaining cancer tissue, while a later increase is suggestive for cancer recurrence. Both, early and late increased postoperative CEA levels are associated with decreased survival (Yakabe et al, 2010; Lin et al, 2011). However, it is unknown whether elevated preoperative CEA levels among rectal cancer patients represents an independent prognostic marker for overall survival.

Therefore, the objective of the present study was to evaluate whether an elevated preoperative CEA level represents an independent prognostic factor in a sample of exclusively rectal cancer patients. The necessity of a focused analysis on a homogenous sample of rectal cancer patients is accentuated as current curative treatment strategies substantially differ between colon and rectal cancer (Lin et al, 2011; NCCN, 2012). To increase statistical power and to further elaborate the possible prognostic impact of CEA in a large cohort of exclusively stage I-III rectal cancer patients, both Cox proportional hazard regression analyses as well as propensity-scoring methods were applied.

\section{MATERIALS AND METHODS}

The present study is based on the colorectal database of the Kantonsspital St Gallen, one of the largest tertiary care centres in Switzerland. Overall, 746 patients with histologically proven rectal cancer were identified from February 1994 to December 
746 consecutive patients with resection of primary rectal cancer (1994-2008)

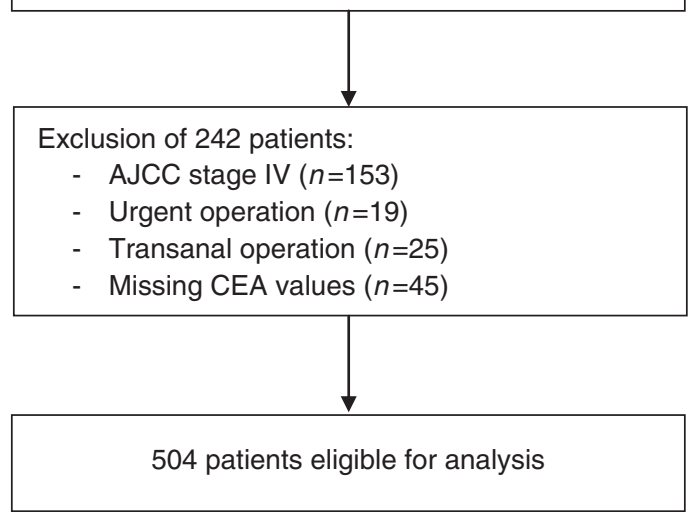

Figure I Patient selection.

2008. Of these patients, 153 were excluded because of the presence of distant metastases (synchronous metastases) at the time of operation (American Joint Committee on Cancer (AJCC) stage IV), 19 patients due to urgent operation, 25 patients because tumour resection was performed transanally (5 of them with missing preoperative CEA levels) and 45 patients due to missing preoperative CEA levels. Figure 1 contains the patient selection flowchart. A total of 504 patients remained for further analyses. Patients were grouped into two groups according to preoperative CEA levels; C0-stage: patients with normal CEA levels $\left(<5 \mathrm{ng} \mathrm{ml}^{-1}\right)$, C1-stage: patients with elevated CEA levels ( $\geqslant 5 \mathrm{ng} \mathrm{ml}^{-1}$; Locker et al, 2006). Carcinoembryonic antigen levels were determined uniformly the day before surgery in all patients. Therefore, in patients undergoing neoadjuvant radiochemotherapy, the CEA levels were measured post neoadjuvant radiochemotherapy.

\section{Operative technique}

The technique for surgical dissection has been earlier described in detail (Zaheer et al, 1998). The type of reconstruction was determined by the operating surgeon depending on tumour and anatomic circumstances. As a standardised procedure, anastomoses were performed after mobilisation of the splenic flexure in a double-stapled technique (Knight and Griffen, 1980). An air leak test was routinely performed by transanal insufflation of air with the anastomosis immersed in saline solution.

\section{Data collection and definitions}

Data on patients demographics, operative details, American Society of Anesthesiologists (ASA) score, cardiovascular disease, postoperative mortality, intra- and postoperative morbidity and histological results were gathered in an electronic database (access, Microsoft). Tumour height, defined as the distance between tumour and anal verge, was based on results of rigid rectosigmoidoscopy, endorectal sonography, MRI scans and/or colonoscopy and was considered in this rank order. Operation time was ascertained from the operation protocol. All patients had histologically proven adenocarcinoma of the rectum; in six patients two rectal cancers were simultaneously found. Neoadjuvant (radio) chemotherapy was routinely performed in patients with cT3/4 and/or $\mathrm{cN}+$ tumours since 2004 according to the interdisciplinary tumour board decision (Sauer et al, 2004). According to the seventh edition of the AJCC, patients with a complete pathological response were included in stage I (AJCC, 2010). The indication for adjuvant treatment was based on the initial clinical stage (before the start of neoadjuvant radiochemotherapy), not on the final pathological disease stage (Sauer et al, 2004).

\section{Follow-up}

Starting 1 June 2007, the follow-up guidelines were as follows: clinical examination and CEA level measurement were obtained every 3 months during the first year and every 6 months for the second and third year after surgery. For the fourth and fifth year postsurgery, an annual clinical examination and CEA level measurement were performed. Moreover, a colonoscopy was done 1 and 4 years postoperatively. Thoraco-abdominal CT scans were performed yearly for 5 years, as well as recto-sigmoidoscopy and endorectal sonography every 6 months during the first 2 years (SGGSSG, 2007).

Before 1 June 2007, follow-up differed mainly in respect to the interval of the clinical examination and CEA level measurements, which were obtained semi-annually for the first 2 years and yearly thereafter for the third to fifth year postoperatively. Also, only one colonoscopy 3 years after resection was performed. Furthermore, either a liver sonography or an abdominal CT scan were performed annually during 5 years to rule out liver metastases in addition to a conventional yearly chest X-ray to detect lung metastases. Finally, recto-sigmoidoscopy was performed semi-annually during the first 2 years.

\section{Statistical analysis}

Statistical analyses were performed using the $\mathrm{R}$ statistical software (www.r-project.org). A two-sided $P$-value $<0.05$ was considered statistically significant. Continuous data are expressed as mean \pm standard deviation. For comparing proportions and continuous variables, $\chi^{2}$ statistics and Mann-Whitney $U$ tests were used, respectively.

First, CEA was assessed as a prognostic factor for overall survival in Cox regression analysis with and without risk-adjustment including a backward variable selection procedure based on the Akaike's information criterion. The risk set included age, gender, body mass index, ASA classification, pre-existing cardiovascular disease, chronic obstructive pulmonary disease and kidney disease, AJCC tumour stage, distance to anal verge, neoadjuvant therapy, type of operation, resection status ( $\mathrm{R} 0 v s \mathrm{R} 1)$, operation time and adjuvant chemotherapy/chemoradiotherapy. A frailty term was included in the Cox regressions to adjust for hidden bias from unobserved variables (Govindarajulu et al, 2011).

Secondly, bipartite matched propensity score analyses as an additional, superior statistical method of adjusting for potential baseline confounding (Rosenbaum, 1985; Rubin, 1997; Boonpipattanapong and Chewatanakornkul, 2006; Fueglistaler et al, 2007) was used. The 'MatchIt' $\mathrm{R}$ package was used to match patients with a similar estimated propensity for C1-stage by simultaneously subclassifying and weighting them (Hansen and Klopfer Olsen, 2006). Afterwards, the baseline risk profiles of the matched patients were compared to assure that no major difference in baseline patient characteristics existed. Finally, the prognostic value of CEA for overall mortality was assessed in a Cox regression analysis with a frailty term for the subclasses and the weights of the bipartite matching propensity score analysis. The analysis was then repeated to assess disease-free survival (excluding four patients with a R1-resection).

\section{Sensitivity analyses}

Finally, various sensitivity analyses were performed. To exclude a potential bias caused by changes in staging procedures, subgroup analyses limited to patients operated before and after 2000 were performed. To adjust for the potential influence of a neoadjuvant therapy on the preoperative serum CEA levels, the prognostic value of CEA was assessed in subgroups of patients with and 
without neoadjuvant treatment. Finally, a sensitivity analysis including 20 patients with transanally resected tumours and available preoperative CEA measurements was performed.

\section{RESULTS}

\section{Patient characteristics and bias estimation}

A total of 504 patients with histologically proven adenocarcinoma of the rectum with preoperative CEA measurement were included in the present analysis. Eleven patients had a complete patho- logical response and were included in stage I. Patients had a median follow-up time of 38.5 months ranging from 1 to 129 months (mean follow-up: 54.7 months). Mean CEA level was $5.8 \pm 16.7 \mathrm{ng} \mathrm{ml}^{-1}$ with a range from 0.5 to $220 \mathrm{ng} \mathrm{ml}^{-1}$. A total of $391(77.6 \%)$ patients had a C0 stage (defined as preoperative CEA $<5 \mathrm{ng} \mathrm{ml}^{-1}$ ) and 113 patients $(22.4 \%)$ had a C1-stage (defined as preoperative CEA $\geqslant 5 \mathrm{ng} \mathrm{ml}^{-1}$ ). Table 1 summarises the characteristics of the patients with $\mathrm{C} 0$-stage and $\mathrm{C} 1$-stage: In univariate analysis, patients with $\mathrm{C} 1$-stage were significantly older, had a higher ASA classification and AJCC stage, had more often a pre-existing cardiovascular disease, underwent less often neoadjuvant treatment, were less often operated with anterior

Table I Patient characteristics and potential bias for carcinoembryonic antigen elevation

\begin{tabular}{|c|c|c|c|c|c|c|c|c|c|c|}
\hline & \multicolumn{4}{|c|}{$\begin{array}{l}\text { Univariate } \\
\text { analysis }\end{array}$} & \multicolumn{2}{|c|}{$\begin{array}{l}\text { Logistic regression } \\
\text { for C-stage }\end{array}$} & \multicolumn{2}{|c|}{$\begin{array}{l}\text { Variable selection } \\
\text { logistic regression } \\
\text { for C-stage }\end{array}$} & \multicolumn{2}{|c|}{$\begin{array}{l}\text { Potential bias after } \\
\text { weighted propensity } \\
\text { score matching }\end{array}$} \\
\hline & $\begin{array}{c}\text { Total } \\
(N=504)\end{array}$ & $\begin{array}{l}\text { C-stage I } \\
(N=\mid 13)\end{array}$ & $\begin{array}{l}\text { C-stage } 0 \\
(N=391)\end{array}$ & $P$ & $\begin{array}{c}\text { OR } \\
(95 \% \mathrm{CI})\end{array}$ & $P^{\mathbf{a}}$ & $\begin{array}{c}\text { OR } \\
(95 \% \mathrm{Cl})\end{array}$ & $P^{\mathbf{a}}$ & $\begin{array}{l}\text { Persisting } \\
\text { bias }^{\text {b }}\end{array}$ & $P$ \\
\hline $\begin{array}{l}\text { Confounding variables } \\
\text { Age (years) }\end{array}$ & $64.9 \pm 12.0$ & $68.7 \pm 11.8$ & $64.0 \pm 12.0$ & $<0.001^{c}$ & 1.01 (0.99-1.03) & 0.407 & - & & 0.031 & $0.773^{c}$ \\
\hline $\begin{array}{l}\text { Gender } \\
\text { Male } \\
\text { Female }\end{array}$ & $\begin{array}{l}320(63.5 \%) \\
184(36.5 \%)\end{array}$ & $\begin{array}{l}74(65.5 \%) \\
39(34.5 \%)\end{array}$ & $\begin{array}{l}246(62.9 \%) \\
145(37.1 \%)\end{array}$ & $0.617^{d}$ & $\begin{array}{c}\text { Reference } \\
0.93(0.57-|.5|)\end{array}$ & 0.773 & - & & $\begin{array}{c}\text { Reference } \\
0.033\end{array}$ & $0.751^{d}$ \\
\hline Body mass index $\left(\mathrm{kg} \mathrm{m}^{-2}\right)$ & $25.6 \pm 4.2$ & $25.0 \pm 4.3$ & $25.8 \pm 4.2$ & $0.080^{c}$ & $0.96(0.90-1.01)$ & 0.108 & $0.96(0.91-1.01)$ & 0.119 & -0.114 & $0.277^{c}$ \\
\hline $\begin{array}{l}\text { ASA stage } \\
\text { II } \\
\text { III/IV }\end{array}$ & $\begin{array}{l}392(77.8 \%) \\
112(22.2 \%)\end{array}$ & $\begin{array}{l}68(60.2 \%) \\
45(39.8 \%)\end{array}$ & $\begin{array}{r}324(82.9 \%) \\
67(17.1 \%)\end{array}$ & $<0.001^{d}$ & $\begin{array}{c}\text { Reference } \\
2.06(1.15-3.70)\end{array}$ & 0.015 & $\begin{array}{c}\text { Reference } \\
2.13(1.22-3.72)\end{array}$ & 0.008 & $\begin{array}{c}\text { Reference } \\
-0.023\end{array}$ & $0.831^{d}$ \\
\hline $\begin{array}{l}\text { Cardiovascular disease } \\
\text { No } \\
\text { Yes }\end{array}$ & $\begin{array}{r}421(83.5 \%) \\
83(16.5 \%)\end{array}$ & $\begin{array}{l}81(71.7 \%) \\
32(28.3 \%)\end{array}$ & $\begin{array}{r}340(87.0 \%) \\
51(13.0 \%)\end{array}$ & $<0.001^{d}$ & $\begin{array}{c}\text { Reference } \\
1.64(0.86-3.08)\end{array}$ & 0.133 & $\begin{array}{c}\text { Reference } \\
\text { I.7। }(0.92-3.16)\end{array}$ & 0.087 & $\begin{array}{c}\text { Reference } \\
-0.023\end{array}$ & $0.828^{d}$ \\
\hline $\begin{array}{l}\text { Chronic obstructive pulmon } \\
\text { No } \\
\text { Yes }\end{array}$ & $\begin{array}{l}\text { ary disease } \\
454(90.1 \%) \\
50(9.9 \%)\end{array}$ & $\begin{array}{l}98(86.7 \%) \\
15(13.3 \%)\end{array}$ & $\begin{array}{c}356(91.0 \%) \\
35(9.0 \%)\end{array}$ & $0.176^{d}$ & $\begin{array}{c}\text { Reference } \\
0.85(0.39-1.77)\end{array}$ & 0.664 & - & & $\begin{array}{c}\text { Reference } \\
0.123\end{array}$ & $0.192^{d}$ \\
\hline $\begin{array}{l}\text { Chronic kidney disease } \\
\text { No } \\
\text { Yes }\end{array}$ & $\begin{aligned} 487 & (96.6 \%) \\
17 & (3.4 \%)\end{aligned}$ & $\begin{aligned} 107 & (94.7 \%) \\
6 & (5.3 \%)\end{aligned}$ & $\begin{array}{c}380(97.2 \%) \\
\quad \text { I | (2.8\%) }\end{array}$ & $0.195^{d}$ & $\begin{array}{c}\text { Reference } \\
0.97(0.28-3.04)\end{array}$ & 0.955 & - & & $\begin{array}{c}\text { Reference } \\
0.082\end{array}$ & $0.371^{d}$ \\
\hline $\begin{array}{l}\text { AJCC stage } \\
\mid \\
\| \\
\|\|\end{array}$ & $\begin{array}{l}185(36.7 \%) \\
149(29.6 \%) \\
170(33.7 \%)\end{array}$ & $\begin{array}{l}21(18.6 \%) \\
37(32.7 \%) \\
55(48.7 \%)\end{array}$ & $\begin{array}{l}\text { I } 64(41.9 \%) \\
112(28.6 \%) \\
115(29.4 \%)\end{array}$ & $<0.001^{d}$ & $\begin{array}{c}\text { Reference } \\
2.54(1.36-4.86) \\
4.32(2.31-8.30)\end{array}$ & 0 & $\begin{array}{c}\text { Reference } \\
2.62(1.40-4.90) \\
4.48(2.40-8.38)\end{array}$ & $<0.001$ & $\begin{array}{c}\text { Reference } \\
-0.001 \\
0.060\end{array}$ & $0.764^{d}$ \\
\hline Distance anal verge $(\mathrm{cm})$ & $7.7 \pm 3.9$ & $7.8 \pm 4.2$ & $7.6 \pm 3.9$ & $0.644^{c}$ & $1.02(0.95-1.09)$ & 0.666 & - & & -0.077 & $0.477^{c}$ \\
\hline $\begin{array}{l}\text { Neoadjuvant therapy } \\
\text { No } \\
\text { Yes }\end{array}$ & $\begin{array}{ll}283 & (56.2 \%) \\
221 & (43.8 \%)\end{array}$ & $\begin{array}{l}77(68.1 \%) \\
36(31.9 \%)\end{array}$ & $\begin{array}{l}206(52.7 \%) \\
185(47.3 \%)\end{array}$ & $0.004^{d}$ & $\begin{array}{c}\text { Reference } \\
0.56(0.32-0.95)\end{array}$ & 0.032 & $\begin{array}{c}\text { Reference } \\
0.50(0.31-0.82)\end{array}$ & 0.005 & $\begin{array}{l}\text { Reference } \\
-0.010\end{array}$ & $0.923 d$ \\
\hline $\begin{array}{l}\text { Operation } \\
\text { Anterior resection } \\
\text { Abdominal perineal } \\
\text { excision }\end{array}$ & $\begin{array}{r}453(89.9 \%) \\
51(10.1 \%)\end{array}$ & $\begin{array}{l}94(83.2 \%) \\
19(16.8 \%)\end{array}$ & $\begin{array}{c}359(91.8 \%) \\
32(8.2 \%)\end{array}$ & $0.007^{d}$ & $\begin{array}{c}\text { Reference } \\
2.15(0.98-4.68)\end{array}$ & 0.056 & $\begin{array}{c}\text { Reference } \\
1.97(1.00-3.84)\end{array}$ & 0.048 & $\begin{array}{l}\text { Reference } \\
-0.028\end{array}$ & $0.796^{d}$ \\
\hline Operation time (hours) & $3.1 \pm 1.2$ & $3.0 \pm 1.0$ & $3.0 \pm 1.3$ & $0.542^{c}$ & $0.98(0.80-1.20)$ & 0.884 & - & & 0.061 & $0.583^{c}$ \\
\hline $\begin{array}{l}\text { Resection } \\
\text { R0 } \\
\text { RI }\end{array}$ & $\begin{array}{c}500(99.2 \%) \\
4(0.8 \%)\end{array}$ & $\begin{array}{c}112 \text { (99.1\%) } \\
\text { I }(0.9 \%)\end{array}$ & $\begin{array}{c}338(99.2 \%) \\
3(0.8 \%)\end{array}$ & $0.901^{d}$ & $\begin{array}{c}\text { Reference } \\
0.69(0.03-7.40)\end{array}$ & 0.774 & - & & $\begin{array}{l}\text { Reference } \\
-0.014\end{array}$ & $0.898^{d}$ \\
\hline $\begin{array}{l}\text { Adjuvant therapy } \\
\text { No } \\
\text { Yes }\end{array}$ & $\begin{array}{l}333(66.1 \%) \\
17 \mid(33.9 \%)\end{array}$ & $\begin{array}{l}79(69.9 \%) \\
34(30.1 \%)\end{array}$ & $\begin{array}{l}254(65.0 \%) \\
137(35.0 \%)\end{array}$ & $0.328^{d}$ & $\begin{array}{c}\text { Reference } \\
0.70(0.40-1.23)\end{array}$ & 0.22 & $\begin{array}{c}\text { Reference } \\
0.66(0.39-1.12)\end{array}$ & 0.126 & $\begin{array}{l}\text { Reference } \\
-0.018\end{array}$ & $0.865^{d}$ \\
\hline $\begin{array}{l}\text { Outcome variables } \\
\text { ICU postoperative } \\
\text { No } \\
\text { Yes }\end{array}$ & $\begin{array}{l}309(61.3 \%) \\
195(38.7 \%)\end{array}$ & $\begin{array}{l}55(48.7 \%) \\
58(51.3 \%)\end{array}$ & $\begin{array}{l}254(65.0 \%) \\
137(35.0 \%)\end{array}$ & $0.002^{d}$ & & & - & & & \\
\hline Hospital stay (days) & $23.5 \pm 13.5$ & $24.4 \pm 13.8$ & $22.8 \pm 13.2$ & $0.416^{c}$ & & & - & & & \\
\hline
\end{tabular}

Abbreviations: $\mathrm{AJCC}=$ American Joint Committee on Cancer; ASA = American Society of Anesthesiologists. $n$ (\%); mean \pm s.d. Logistic regression (full model and backward

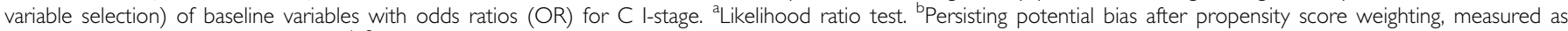
standardised mean difference. ${ }^{c}$ t-test. ${ }^{\mathrm{d}} \chi^{2}$-test. 
Table 2 Prognostic factors for overall mortality

\begin{tabular}{|c|c|c|c|c|c|c|}
\hline & \multicolumn{2}{|c|}{ Unadjusted $^{a}$} & \multicolumn{2}{|c|}{ Cox regression, full model ${ }^{b}$} & \multicolumn{2}{|c|}{ Cox regression, variable selection ${ }^{c}$} \\
\hline $\begin{array}{l}\text { C-stage } \\
\text { C-stage } 0 \\
\text { C-stage I }\end{array}$ & $\begin{array}{c}\text { Reference } \\
2.80(1.95-4.02)\end{array}$ & $<0.001$ & $\begin{array}{c}\text { Reference } \\
1.9 \mid(1.28-2.85)\end{array}$ & 0.001 & $\begin{array}{c}\text { Reference } \\
1.98(1.36-2.90)\end{array}$ & $<0.001$ \\
\hline Age (years) & $1.08(1.06-1.10)$ & $<0.001$ & $1.07(1.05-1.09)$ & 0.000 & $1.07(1.05-1.09)$ & $<0.001$ \\
\hline$B M I\left(\mathrm{~kg} \mathrm{~m}^{-2}\right)$ & $0.93(0.89-0.97)$ & 0.001 & $0.94(0.89-0.99)$ & 0.010 & $0.94(0.89-0.98)$ & 0.006 \\
\hline $\begin{array}{l}\text { ASA stage } \\
\| \\
\| / I / I V\end{array}$ & $\begin{array}{c}\text { Reference } \\
2.58(1.79-3.73)\end{array}$ & $<0.001$ & $\begin{array}{c}\text { Reference } \\
1.22(0.77-1.95)\end{array}$ & 0.235 & - & \\
\hline $\begin{array}{l}\text { Chronic obstructive pulmon } \\
\text { No } \\
\text { Yes }\end{array}$ & $\begin{array}{l}\text { sease } \\
\text { Reference } \\
2.21(1.36-3.60)\end{array}$ & 0.003 & $\begin{array}{c}\text { Reference } \\
1.03(0.60-1.78)\end{array}$ & 0.553 & - & \\
\hline $\begin{array}{l}\text { Chronic kidney disease } \\
\text { No } \\
\text { Yes }\end{array}$ & $\begin{array}{c}\text { Reference } \\
4.04(1.90-8.62)\end{array}$ & 0.002 & $\begin{array}{c}\text { Reference } \\
2.01(0.85-4.74)\end{array}$ & 0.106 & $\begin{array}{c}\text { Reference } \\
2.28(1.03-5.04)\end{array}$ & 0.049 \\
\hline $\begin{array}{l}\text { AJCC stage } \\
\text { I } \\
\| \\
\text { III }\end{array}$ & $\begin{array}{c}\text { Reference } \\
1.80(1.11-2.91) \\
2.66(1.70-4.17)\end{array}$ & $<0.001$ & $\begin{array}{c}\text { Reference } \\
1.36(0.81-2.28) \\
2.12(1.29-3.49)\end{array}$ & 0.006 & $\begin{array}{c}\text { Reference } \\
1.41(0.85-2.32) \\
2.08(1.31-3.31)\end{array}$ & 0.004 \\
\hline $\begin{array}{l}\text { Distance to anal verge } \\
\qquad 10 \mathrm{~cm} \\
>5-10 \mathrm{~cm} \\
0-5 \mathrm{~cm}\end{array}$ & $\begin{array}{c}\text { Reference } \\
1.41(0.85-2.33) \\
2.38(1.45-3.92)\end{array}$ & 0.001 & $\begin{array}{c}\text { Reference } \\
1.52(0.88-2.63) \\
1.97(1.04-3.76)\end{array}$ & 0.064 & $\begin{array}{c}\text { Reference } \\
1.48(0.88-2.49) \\
1.93(1.11-3.37)\end{array}$ & 0.039 \\
\hline Operation time (hours) & $1.02(0.90-1.17)$ & 0.637 & $1.02(0.88-1.19)$ & 0.612 & - & \\
\hline $\begin{array}{l}\text { Resection } \\
\text { RO } \\
\text { RI }\end{array}$ & $\begin{array}{c}\text { Reference } \\
5.11(1.51-17.3)\end{array}$ & 0.027 & $\begin{array}{c}\text { Reference } \\
5.48(1.50-20.0)\end{array}$ & 0.023 & $\begin{array}{c}\text { Reference } \\
5.65(1.59-20.1)\end{array}$ & 0.005 \\
\hline $\begin{array}{l}\text { Adjuvant therapy } \\
\text { No } \\
\text { Yes }\end{array}$ & $\begin{array}{c}\text { Reference } \\
0.67(0.45-0.99)\end{array}$ & 0.037 & $\begin{array}{c}\text { Reference } \\
0.96(0.60-1.54)\end{array}$ & 0.615 & - & \\
\hline
\end{tabular}

Abbreviations: $A J C C=$ American Joint Committee on Cancer; ASA = American Society of Anesthesiologists. Hazard ratios (HR) with $95 \%$ confidence intervals resulting from: a Univariate, Cox proportional hazards regression analysis for overall mortality; ' Independent, full model Cox proportional hazards regression analysis for overall mortality; Independent, backward variable selection Cox proportional hazards regression analysis for overall mortality (confirmed by forward variable selection with identical results);

${ }^{\mathrm{d} P}$-values from likelihood ratio tests.

resection with primary anastomosis and were more often transferred to the ICU postoperatively.

\section{C-stage as a prognostic factor for overall and disease-free survival}

An unadjusted Cox proportional hazards regression analysis revealed C1-stage as a statistically significant prognostic factor with an approximately $180 \%$ increased risk of mortality (hazard ratio
(HR) of death $=2.80,95 \%$ confidence interval $(\mathrm{CI})=1.95-4.02$, $P<0.001$ ) (Table 2). The 5-year survival for patients with C1-stage was $46.7 \%$ (95\% CI: 37.1-59.0\%) compared with $81.0 \%$ (95\% CI: 76.3-85.9\%) for rectal cancer patients with $\mathrm{C} 0$-stage (Figure 2). In unadjusted Cox regression analyses, overall mortality was in addition to C1-stage dependent on age, body mass index, preexisting cardiovascular disease, chronic obstructive pulmonary disease, chronic kidney disease, ASA stage, AJCC stage, distance to anal verge, neoadjuvant treatment, adjuvant therapy, R0 vs R1 


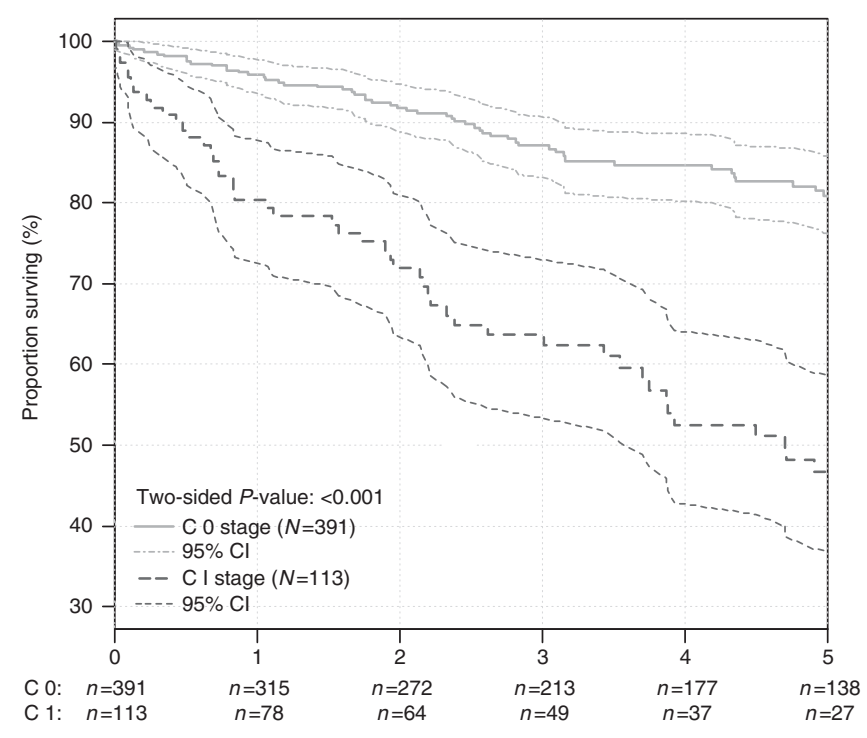

Years after operation/patients at risk in C-stage 0 and I

Figure 2 Kaplan-Meier curve for overall survival in unmatched analysis. Life tables for the number of rectal adenocarcinoma patients at risk are given below each plot. Survival curves are provided with 95\% point-wise confidence intervals.

resection and type of operation (Table 2). After risk-adjusting the Cox regression analysis, C1-stage remained associated with an increased risk for overall mortality of $91 \%$ (HR of death $=1.91$, $95 \% \mathrm{CI}=1.28-2.85, P=0.001)$. In a backward variable selection, C1-stage (HR of death $=1.98,95 \% \mathrm{CI}=1.36-2.90, P<0.001)$ was confirmed as an independent predictor for increased overall mortality together with age, body mass index, chronic kidney disease, AJCC stage, R1 vs R0 resection and tumour distance from the anal verge. A forward variable selection yielded the same independent predictors.

C-stage also served as a prognostic factor for disease-free survival. An unadjusted Cox proportional hazards regression analysis revealed C1-stage as a statistically significant prognostic factor with an approximately $163 \%$ increased risk for recurrence (HR of recurrence: 2.63 , 95\% CI: 1.89-3.65, P<0.001) (Table 3). After risk-adjusting in Cox regression analysis, C1-stage remained associated with an increased risk for recurrence of $117 \%$ (HR of recurrence: $2.17,95 \%$ CI: $1.51-3.10, P<0.001)$. In a backward variable selection, C1-stage (HR of recurrence: $2.08,95 \% \mathrm{CI}$ : 1.48-2.94, $P<0.001)$ was confirmed as an independent predictor for disease-free survival. The 5-year disease-free survival for patients with C1-stage was 38.9\% (95\% CI: 29.9-50.7\%) compared with $72.3 \%$ (95\% CI: $67.2-77.8 \%$ ) for rectal cancer patients with C0-stage (Figure 3).

\section{Adjusting for patients characteristics with propensity score matching}

To further corroborate the interesting findings from univariate and Cox proportional hazard regression analyses, the propensity score was estimated incorporating the patient characteristics listed in Table 1 . The propensity for C1-stage was $0.33 \pm 0.17$ compared with $0.19 \pm 0.14$ for patients with $\mathrm{C} 0$-stage $(P<0.001)$. After performing the propensity score-matching procedure, the score was practically identical for patients with $\mathrm{C} 1$-stage and $\mathrm{C} 0$-stage $(0.33 \pm 0.17$ vs $0.33 \pm 0.17, P=0.928)$. Figure 4 displays the distribution of the propensity score before and after matching for patients with C1-stage and C0-stage. The right columns of Table 1 summarise the persisting bias between the characteristics of patients with $\mathrm{C} 1$-stage and $\mathrm{C} 0$-stage after performing the propensity score-matching analysis. No significant differences in patient characteristics were found any more between C1-stage and $\mathrm{C} 0$-stage patients (Table 1), thus precluding bias of the observed variables.

\section{Propensity score-matched prognostic factors for overall and disease-free survival}

When performing a Cox regression analysis after the propensity score-matching procedure, C1-stage was a persistently significant predictor for overall mortality $(\mathrm{HR}=2.05,95 \% \mathrm{CI}=1.47-2.86$, $P<0.001)$. In the propensity score-matched analysis, the 5-year survival for patients with C1-stage was $46.7 \% \quad(95 \%$ CI: 37.1-59.0\%) compared with 71.5\% (95\% CI: 66.0-77.5\%) for C0stage patients $(P<0.001)$ (Figure 5).

When performing a Cox regression analysis after a propensity score-matching procedure, C1-stage remained a significant predictor for disease-free survival (HR: 1.90, 95\% CI: 1.40-2.57, $P<0.001)$. In the propensity score-matched analysis, the 5-year disease-free survival for patients with C1-stage was 38.9\% (95\% CI: 29.9-50.7\%) compared with $62.8 \%$ (95\% CI: $57.2-69.0 \%)$ for C0-stage patients $(P<0.001)$ (Figure 6).

\section{Sensitivity analyses}

In the present investigation, in $18(13.5 \%)$ out of 133 patients who died, the cause of death remained unclear as no autopsy was performed, in 53 patients (39.9\%) death was tumour related and in 62 patients $(46.6 \%)$ death was not tumour related. In univariate analysis, the HR for cancer-specific survival was 2.33 (95\% CI: 1.28-4.24, $P=0.007$ ) for C1-stage (elevated preoperative CEA level). In multivariable analysis after adjusting for AJCC stage, age, type of operation, distance of the tumour from the anal verge and R0 vs R1 resection, the HR for cancer-specific survival was 2.40 (95\% CI: 1.26 to $4.59, P=0.008$ ) for preoperative C1-stage.

In a subgroup analysis limited to the 323 patients with available data for postoperative CEA measurements, the preoperative C1-stage (HR: $1.86 ; 95 \%$ CI: $1.02-3.36, P=0.023$ ), as well as the postoperative C1-stage (HR: 2.33; 95\% CI: $1.01-5.41, P=0.038$ ) were independent predictors for mortality in adjusted Coxregression analysis.

In an additional subgroup analysis limited to 221 patients receiving neoadjuvant therapy, $\mathrm{C} 1$-stage did significantly increase the risk of mortality (HR: 2.59 ; $95 \% \mathrm{CI}: 1.25-5.33, P=0.014$ ). The risk of mortality (HR: 1.65 ; 95\% CI: $1.05-2.58, P=0.033$ ) in patients classified as C1-stage who did not receive neoadjuvant therapy was also significantly increased.

To address the potential influence of changing staging routines throughout the study period, a sensitivity analysis was performed in the subsets of patients operated prior and after the year 2000. In the subgroup analysis of 211 patients operated before the year 2000, C1-stage significantly increased the risk of mortality (HR: 1.72; 95\% CI: $1.08-2.73, P=0.024)$. When analysing the 293 patients operated from 2000 onwards, C1-stage also significantly increased the risk of mortality (HR: 3.11 ; 95\% CI: $1.70-5.67, P=0.001$ ).

A total of 20 patients with transanally resected tumours and preoperative CEA level could have potentially been included in the present study, only one of them with C1-stage (5\%). When performing a sensitivity analysis including these 20 patients, the results did not change relevantly (overall survival HR: 2.03 for C1-stage (95\% CI: $1.45-2.83, P<0.001$ ); disease-free survival HR: 2.18 for C1-stage (95\% CI: $1.61-2.96, P<0.001)$ ).

\section{DISCUSSION}

The present investigation provides compelling evidence that an elevated preoperative CEA level in stage I-III rectal cancer patients 
Table 3 Prognostic factors for disease-free survival

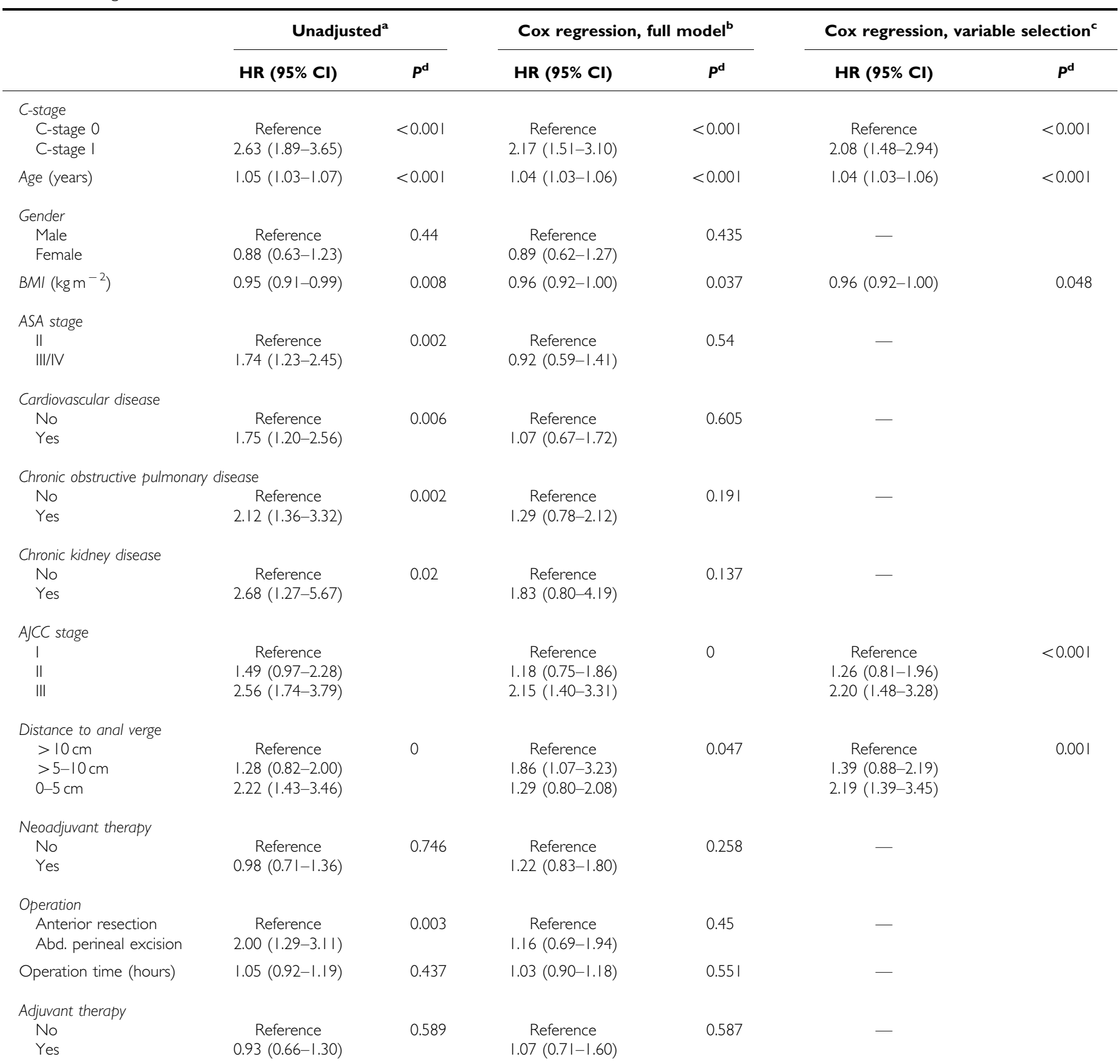

Abbreviations: $\mathrm{AJCC}=$ American Joint Committee on Cancer; ASA = American Society of Anesthesiologists. Hazard ratios (HR) with 95\% confidence intervals resulting from:

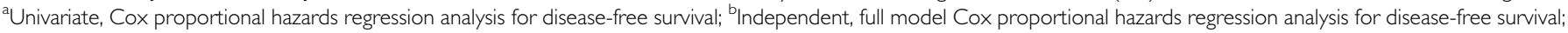
Independent, backward variable selection Cox proportional hazards regression analysis for disease-free survival (confirmed by forward variable selection with identical results);

${ }^{d} P$-values from likelihood ratio tests.

is independently associated with a decreased overall, disease-free and cancer-specific survival. These survival benefits were found to be highly statistically significant in both multivariable Cox proportional hazard regression model as well as in propensity score-matched survival analyses and persistent in various subset analyses. Preoperative measurement of CEA level does provide important prognostic information.

A recent study published in the Journal of National Cancer Institute provides clear evidence that an increased preoperative CEA level is an independent prognostic factor for colon cancer patients (Thirunavukarasu et al, 2011). This population-based study assessed almost 18000 patients from the Surveillance,
Epidemiology and End Results database. C1-stage was found to have a $60 \%$ increased risk of overall mortality, however, the authors were not able to adjust for potentially important covariates like comorbidities and body mass index. Interestingly, in the present investigation on exclusively rectal cancer patients, the point estimate of the HR of overall mortality among C0-stage and C1-stage rectal cancer patients was even higher (HR of 1.98 in Cox proportional analyses), indicating a two-fold increase in risk of dying having a preoperative elevated CEA level. This finding is not only statistically significant but also highly clinically relevant.

The results of this investigation are partly supported by an earlier study performed by Park et al (2006). In contrast to the 


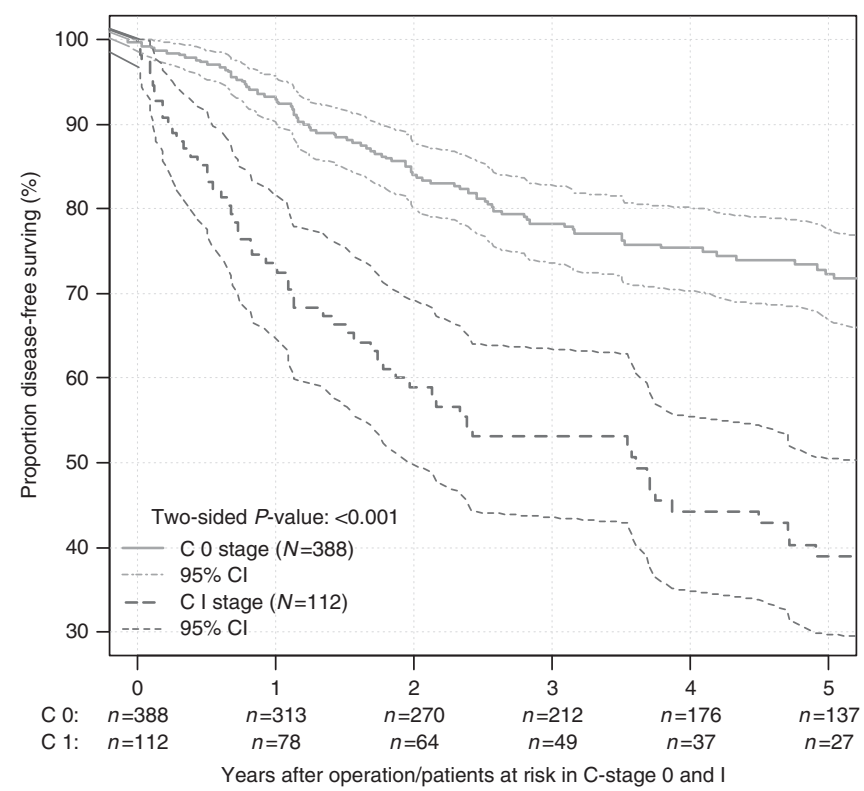

Figure 3 Kaplan-Meier curve for disease-free survival in unmatched analysis. Life tables for the number of rectal adenocarcinoma patients at risk are given below each plot. Survival curves are provided with 95\% point-wise confidence intervals. Analysis limited to 500 patients with R0-resection
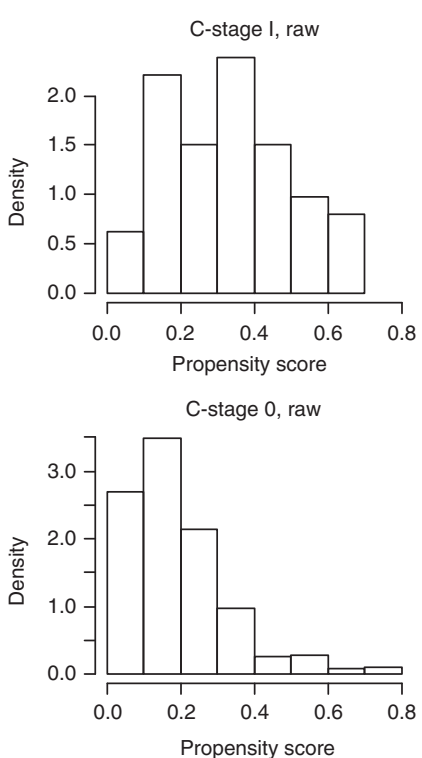

C-stage I, matched

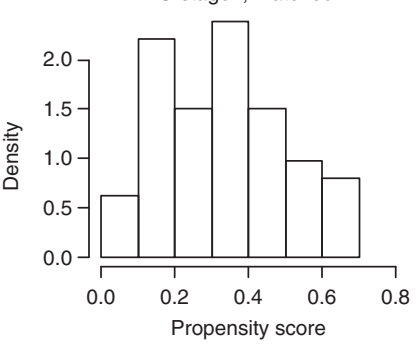

C-stage 0 , matched

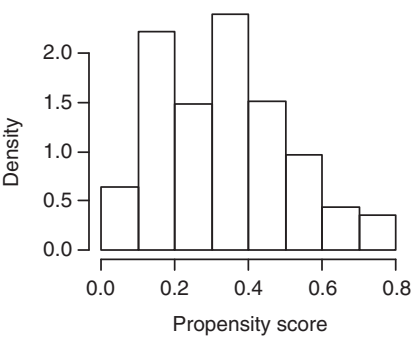

Figure 4 Distribution of propensity score before and after propensity score analysis. Left upper and lower panel show the distribution of the propensity score for patients with $\mathrm{Cl}$-stage and $\mathrm{CO}$-stage before the matching procedure. Right upper and lower panel demonstrate the distribution of the propensity score after full propensity score matching.

present study, they focused on perioperative changes in the serum CEA level measured in the preoperative and early postoperative period for predicting recurrence and survival in stage II and III rectal cancer patients. Park et al reported that stage II and III rectal cancer patients with pre- and postoperative normal CEA levels had a significantly better survival than patients with preand postoperative increased CEA levels (Park et al, 2006). Other studies investigating the influence of preoperative CEA levels were performed in more heterogeneous samples of colorectal cancer

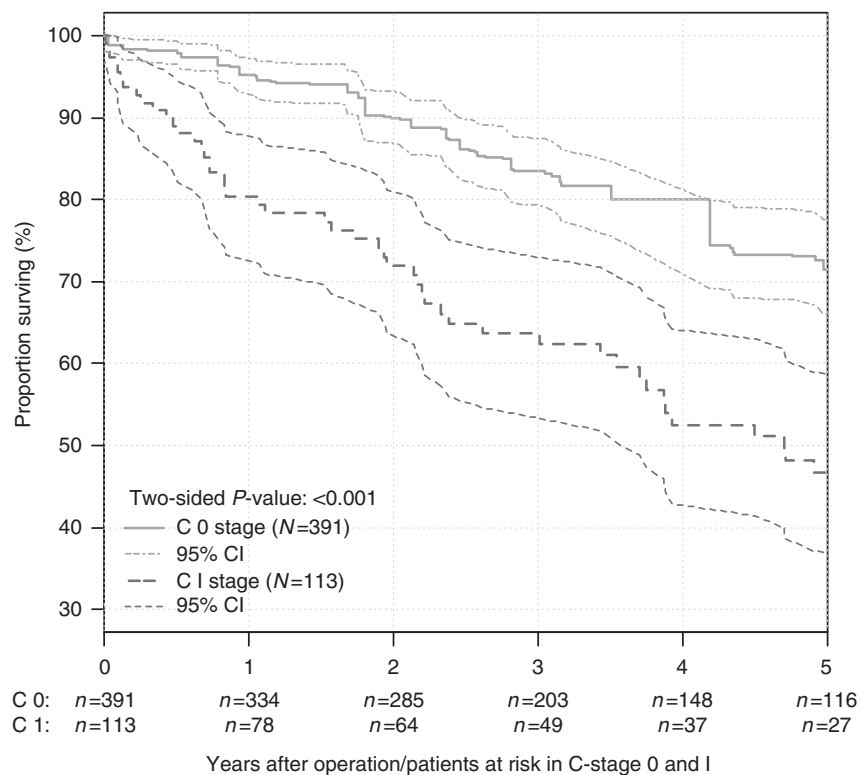

Figure 5 Kaplan-Meier curve for overall survival in propensity scorematched analysis. Life tables for the number of rectal adenocarcinoma patients at risk are given below each plot. Survival curves are provided with $95 \%$ point-wise confidence interval.

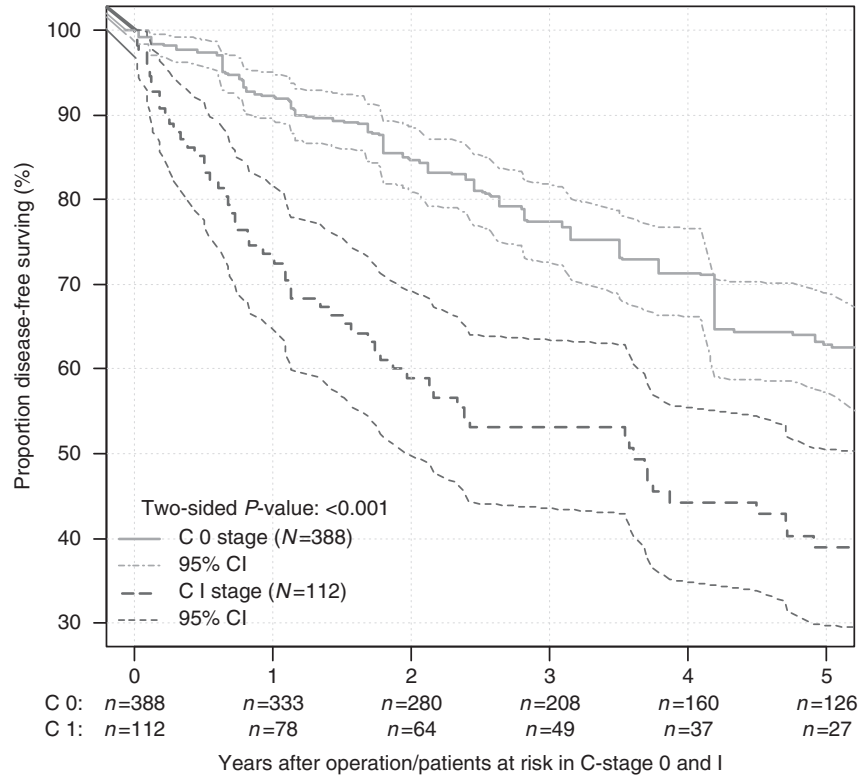

Figure 6 Kaplan-Meier curve for disease-free survival in propensity score-matched analysis. Life tables for the number of rectal adenocarcinoma patients at risk are given below each plot. Survival curves are provided with $95 \%$ point-wise confidence intervals. Analysis limited to 500 patients with $\mathrm{R} 0$-resection.

patients (Chapman et al, 1998; Diez et al, 2000; Takagawa et al, 2008; Sun et al, 2009), which differs from the present investigation involving exclusively rectal cancer patients. Although the authors of these studies concluded that a preoperative elevated CEA level is associated with poorer survival, two recent investigations among colorectal cancer patients did not find any significant association (Li et al, 2009; Lin et al, 2011).

Besides the prognostic capacity of preoperative CEA in colorectal cancer patients, postoperative measurement of CEA levels is 
also a valuable diagnostic tool. A persistently high CEA level or an early elevated postoperative CEA level is associated with precocious relapse while the majority of those tumours recur in the liver (Takagawa et al, 2008; Park et al, 2009; Lin et al, 2011). Suggestions to monitor postoperative CEA levels are not limited to patients with preoperatively elevated CEA but should be performed for all patients according to most guidelines. A secondary analysis of the TME trial of the Dutch ColoRectal Cancer group showed that $41 \%$ of patients with a preoperative normal CEA level developed an increased CEA level $\left(>5 \mathrm{ng} \mathrm{ml}^{-1}\right)$ when they experienced a recurrent disease in the follow-up (Grossmann et al, 2007). Although most studies investigated preor postoperative CEA levels or both, Perez et al (2009) focused on CEA levels after neoadjuvant radiochemotherapy in rectal cancers. In their study, increased levels after neoadjuvant radiochemotherapy in rectal cancer patients was associated with poor survival. However, increased CEA levels before neoadjuvant radiochemotherapy was not associated with 5 -year overall survival but showed decreased disease-free survival.

\section{Limitations}

The present study has several limitations that need to be addressed. First, this is a cohort study and not a randomised controlled trial. However, for the research question at hand, it is impossible to perform a randomised trial, and a cohort study is probably the best suited study design. Second, although extensive risk-adjustment was performed, a potential selection bias cannot fully be excluded with certainty in the present cohort study. However, in Cox proportional hazard regression analyses, propensity score analyses and different subset analyses, the differences in

\section{REFERENCES}

AJCC (2010) In AJCC Cancer Staging Manual, Edge SB, Byrd DR, Compton CC, Fritz AG, Greene FL, Trotti A (eds). 7th edn, Vol X, 646pp, 130 illus. with CD-ROM. ISBN 978-0-387-88440-0. Springer: New York

Boonpipattanapong T, Chewatanakornkul S (2006) Preoperative carcinoembryonic antigen and albumin in predicting survival in patients with colon and rectal carcinomas. J Clin Gastroenterol 40(7): 592-595

Cancer-Statistics US (2010) US Cancer Statistics Working Group. United States Cancer Statistics: 1999-2007 Incidence and Mortality Web-based Report. Atlanta: U.S. Department of Health and Human Services, Centers for Disease Control and Prevention and National Cancer Institute (2010) Available at: www.cdc.gov, last accessed 12 March 2012

Chapman MA, Buckley D, Henson DB, Armitage NC (1998) Preoperative carcinoembryonic antigen is related to tumour stage and long-term survival in colorectal cancer. Br J Cancer 78(10): 1346-1349

Diez M, Pollan M, Muguerza JM, Gaspar MJ, Duce AM, Alvarez MJ, Ratia T, Hernandez P, Ruiz A, Granell J (2000) Time-dependency of the prognostic effect of carcinoembryonic antigen and p53 protein in colorectal adenocarcinoma. Cancer 88(1): 35-41

Fueglistaler P, Adamina M, Guller U (2007) Non-inferiority trials in surgical oncology. Ann Surg Oncol 14(5): 1532-1539

Govindarajulu US, Lin H, Lunetta KL, D'Agostino Sr RB (2011) Frailty models: applications to biomedical and genetic studies. Stat Med 30(22): 2754-2764

Grossmann I, de Bock GH, Meershoek-Klein Kranenbarg WM, van de Velde CJ, Wiggers T (2007) Carcinoembryonic antigen (CEA) measurement during follow-up for rectal carcinoma is useful even if normal levels exist before surgery. A retrospective study of CEA values in the TME trial. Eur J Surg Oncol 33(2): 183-187

Hansen BB, Klopfer Olsen S (2006) Optimal full matching and related designs via network flows. J Computat Graphical Statistics 15(3): 609-627

Howlader N, Noone AM, Krapcho M, Neyman N, Aminou R, Waldron WAltekruse SF, Kosary CL, Ruhl J, Tatalovich Z, Cho H, Mariotto A, Eisner MP, Lewis DR, Chen HS, Feuer EJ, Cronin KA, Edwards BK (2011) (eds). SEER Cancer Statistics Review, 1975-2008. National Cancer Institute: Bethesda, MD. http://seer.cancer.gov/csr/1975_2008/, based on November 2010 SEER data submission, posted to the SEER web site overall survival between patients with normal vs elevated preoperative CEA was not only statistically significant but also clinically relevant. Thus, it is likely that the observed differences are real.

\section{CONCLUSIONS}

The present investigation based on a large cohort of exclusively rectal cancer patients - one of the first in the literature - provides compelling evidence that an elevated preoperative CEA level is a strong and in our study independent predictor of overall survival. The findings of the present study increase the evidence that preoperative CEA measurement should be considered as an additional tool for preoperative tumour staging in addition to the TNM classification in stage I-III rectal cancer patients. A prospective, multicentre evaluation of the combination of preoperative CEA and TNM staging compared with histopathological staging alone might provide the highest evidence possible to further corroborate our findings.

\section{ACKNOWLEDGEMENTS}

This work was supported in part by Grants PBBEP3-131567 (MW) from the Swiss National Science Foundation.

\section{Conflict of interest}

The authors declare no conflict of interest.
Kim JY, Kim NK, Sohn SK, Kim YW, Kim KJ, Hur H, Min BS, Cho CH (2009) Prognostic value of postoperative CEA clearance in rectal cancer patients with high preoperative CEA levels. Ann Surg Oncol 16(10): 2771-2778

Knight CD, Griffen FD (1980) An improved technique for low anterior resection of the rectum using the EEA stapler. Surgery 88(5): 710-714

Li M, Li JY, Zhao AL, He JS, Zhou LX, Li YA, Gu J (2009) Comparison of carcinoembryonic antigen prognostic value in serum and tumour tissue of patients with colorectal cancer. Colorectal Dis 11(3): 276-281

Lin JK, Lin CC, Yang SH, Wang HS, Jiang JK, Lan YT, Lin TC, Li AF, Chen WS, Chang SC (2011) Early postoperative CEA level is a better prognostic indicator than is preoperative CEA level in predicting prognosis of patients with curable colorectal cancer. Int $J$ Colorectal Dis 26(9): 1135-1141

Locker GY, Hamilton S, Harris J, Jessup JM, Kemeny N, Macdonald JS, Somerfield MR, Hayes DF, Bast Jr RC (2006) ASCO 2006 update of recommendations for the use of tumor markers in gastrointestinal cancer. J Clin Oncol 24(33): 5313-5327

NCCN (2012) National Comprehensive Cancer Network (NCCN) guidelines. http://www.nccn.org/index.asp (last accessed 12 March 2012)

Park IJ, Choi GS, Lim KH, Kang BM, Jun SH (2009) Serum carcinoembryonic antigen monitoring after curative resection for colorectal cancer: clinical significance of the preoperative level. Ann Surg Oncol 16(11): 3087-3093

Park YA, Lee KY, Kim NK, Baik SH, Sohn SK, Cho CW (2006) Prognostic effect of perioperative change of serum carcinoembryonic antigen level: a useful tool for detection of systemic recurrence in rectal cancer. Ann Surg Oncol 13(5): 645-650

Parkin DM, Bray F, Ferlay J, Pisani P (2005) Global cancer statistics, 2002. CA Cancer J Clin 55(2): 74-108

Perez RO, Sao Juliao GP, Habr-Gama A, Kiss D, Proscurshim I, Campos FG, Gama-Rodrigues JJ, Cecconello I (2009) The role of carcinoembriogenic antigen in predicting response and survival to neoadjuvant chemoradiotherapy for distal rectal cancer. Dis Colon Rectum 52(6): 1137-1143

Rosenbaum PRaDBR (1985) Constructing a control group using multivariate matched sampling methods that incorporate the propensity score. American Statistician 39(1): 33-38 
Rubin DB (1997) Estimating causal effects from large data sets using propensity scores. Ann Intern Med 127(8 Part 2): 757-763

Sauer R, Becker H, Hohenberger W, Rodel C, Wittekind C, Fietkau R, Martus P, Tschmelitsch J, Hager E, Hess CF, Karstens JH, Liersch T, Schmidberger H, Raab R (2004) Preoperative versus postoperative chemoradiotherapy for rectal cancer. N Engl J Med 351(17): 1731-1740

SGGSSG (2007) Konsensus-Empfehlungen zur Nachsorge nach kurativ operiertem kolorektalem Karzinom. Schweizerische Gesellschaft für Gastroenterologie. http://www.sggssg.ch/Dokumente/Merkblaetter/NaSo_ CRC_d.pdf, last accessed 7 May 2012

Sun LC, Chu KS, Cheng SC, Lu CY, Kuo CH, Hsieh JS, Shih YL, Chang SJ, Wang JY (2009) Preoperative serum carcinoembryonic antigen, albumin and age are supplementary to UICC staging systems in predicting survival for colorectal cancer patients undergoing surgical treatment. BMC Cancer 9: 288

Takagawa R, Fujii S, Ohta M, Nagano Y, Kunisaki C, Yamagishi S, Osada S, Ichikawa Y, Shimada H (2008) Preoperative serum carcinoembryonic antigen level as a predictive factor of recurrence after curative resection of colorectal cancer. Ann Surg Oncol 15(12): 3433-3439

Thirunavukarasu P, Sukumar S, Sathaiah M, Mahan M, Pragatheeshwar KD, Pingpank JF, Zeh 3rd H, Bartels CJ, Lee KK, Bartlett DL (2011) $\mathrm{C}$-stage in colon cancer: implications of carcinoembryonic antigen biomarker in staging, prognosis, and management. J Natl Cancer Inst 103(8): 689-697

Wanebo HJ, Rao B, Pinsky CM, Hoffman RG, Stearns M, Schwartz MK, Oettgen HF (1978) Preoperative carcinoembryonic antigen level as a prognostic indicator in colorectal cancer. $N$ Engl J Med 299(9): 448-451

Yakabe T, Nakafusa Y, Sumi K, Miyoshi A, Kitajima Y, Sato S, Noshiro H, Miyazaki K (2010) Clinical significance of CEA and CA19-9 in postoperative follow-up of colorectal cancer. Ann Surg Oncol 17(9): 2349-2356

Zaheer S, Pemberton JH, Farouk R, Dozois RR, Wolff BG, Ilstrup D (1998) Surgical treatment of adenocarcinoma of the rectum. Ann Surg 227(6): $800-811$

This work is published under the standard license to publish agreement. After 12 months the work will become freely available and the license terms will switch to a Creative Commons Attribution-NonCommercial-Share Alike 3.0 Unported License. 